\title{
BPM SIGNAL CONDITIONING FOR A WIDE RANGE OF SINGLE-BUNCH CURRENT OPERATION IN THE DUKE STORAGE RING*
}

\author{
J. $\mathrm{Li}^{\dagger}$, P. Wang and Y.K. Wu \\ FEL Lab, Duke University, Durham, NC 27708 USA
}

\begin{abstract}
The beam position monitor system of the Duke storage ring has been in operation since 1998. Recently, by injecting at higher energy with a booster synchrotron, the single bunch current threshold is much more increased. The high peak voltage associated with high single-bunch current degrades the performance of the BPM system and can potentially damage the BPM electronics. To improve the accuracy of the orbit measurement, we carefully studied the BPM signal and found a way to overcome this problem. This paper reports our findings and presents a solution to condition the signal for a wide single-bunch current operation.
\end{abstract}

\section{INTRODUCTION}

The beam position monitor (BPM) system of the Duke storage ring has been in operation since 1998 [1] and providing reasonable reliable orbit data for orbit feedback and various user operations at relatively low beam current. Thirty-four BPMs are currently used to measure the closed orbit of electron beam in the Duke storage ring. Among these BPMs, three types of electodes are used as signal pickups:(1) $30.35 \mathrm{~mm}$ long striplines in the north straight section (NSS); (2) $21.64 \mathrm{~mm}$ long striplines in the two arcs; (3) buttons in the south straight section (SSS) and NSS. These pickups are connected to Bergoz BPM electronics modules via RG223 cable. In the electronics module, the four signals from each BPM are time-multiplexed into single one to a superheterodyne receiver. A 1-GHz low pass filter (LPF) in each of the four input channel is used to protect the multiplexer from very short, high voltage pulses. For safety reasons, the signal peak after this LPF should not exceed 5 volts [2].

The electronics module is designed for operating over a frequency range of $178.5 \pm 20 \mathrm{MHz}$ [2], around the frequency of the Duke storage ring RF cavity. The electronics module works from -70 to $5 \mathrm{dBm}$ in this specified frequency range [3].

Strong beam current dependency had been observed and studied. Steps had been taken to reduce this effect [4]. In the 2006, a booster synchrotron was commissioned and electron beam was successfully extracted from it and injected into the storage ring in the Spring of 2007 [5]. The

\footnotetext{
* Work is supported by US AFOSR MFEL grant \#FA9550-04-01-0086.
}

$\dagger$ email: jing@fel.duke, tel\#: (919)660-2657

06 Instrumentation, Controls, Feedback \& Operational Aspects extraction energy of the booster is from $240 \mathrm{MeV}$ to 1.2 $\mathrm{GHz}$. By injecting at a higher energy, the single beam current threshold of the Duke storage ring is much more increased, e.g. $51 \mathrm{~mA}$ single bunch beam was stored in the storage ring at $600 \mathrm{MeV}$ without lasing in March, 2007. The high peak voltage associated with a high single-bunch current degrades the performance of the BPM system and can potentially damage the BPM electronics. To improve the accuracy of the orbit measurement at high single-bunch currents, we carefully studied the BPM signal and found a solution to reduce the peak pickup voltage.

\section{BPM SIGNAL AT DIFFERENT BEAM CURRENT}

The Duke storage ring is required to be operated over a wide range of beam current - for some machine studies, the storage ring need to be operated below $1 \mathrm{~mA}$ in the single bunch mode; during some user operation, the singlebunch current can be as high as $100 \mathrm{~mA}$. This requires the

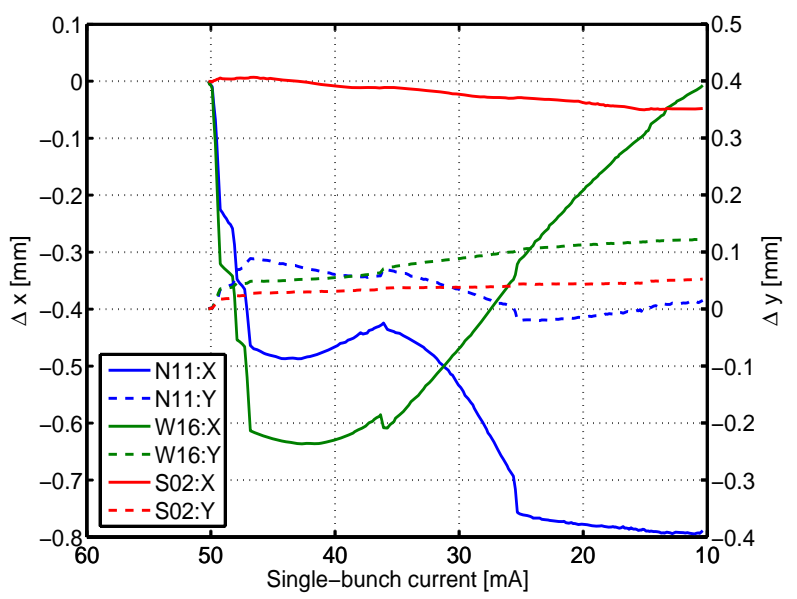

Figure 1: Orbit variations with beam current. N11 is a long stripline BPM; W16 is a short stripline BPM; S02 is a button BPM.

BPM system to work reasonably well in a range from lower than $1 \mathrm{~mA}$ to $100 \mathrm{~mA}$. To test the reliability of the BPM system, we injected $51 \mathrm{~mA}$ of single-bunch beam current into the storage ring and monitored the closed orbit as the beam current decayed. The results indicate, as shown in Fig. 1, the button BPMs, e.g. S02 BPM, work reasonable

T03 Beam Diagnostics and Instrumentation 
well in the current range from $51 \mathrm{~mA}$ to $10 \mathrm{~mA}$; the orbit variations are within $50 \mu \mathrm{m}$ in both directions. The long stripline BPMs, like N11 BPM, can not provide reliable orbit values above $25 \mathrm{~mA}$; they work well below $25 \mathrm{~mA}$. The orbits measured by arc BPMs have big variations in the current range from $51 \mathrm{~mA}$ to $10 \mathrm{~mA}$, which may be caused by the high peak pickup voltage, off-centered orbits at the location of BPM pickups, temperature variations, etc.
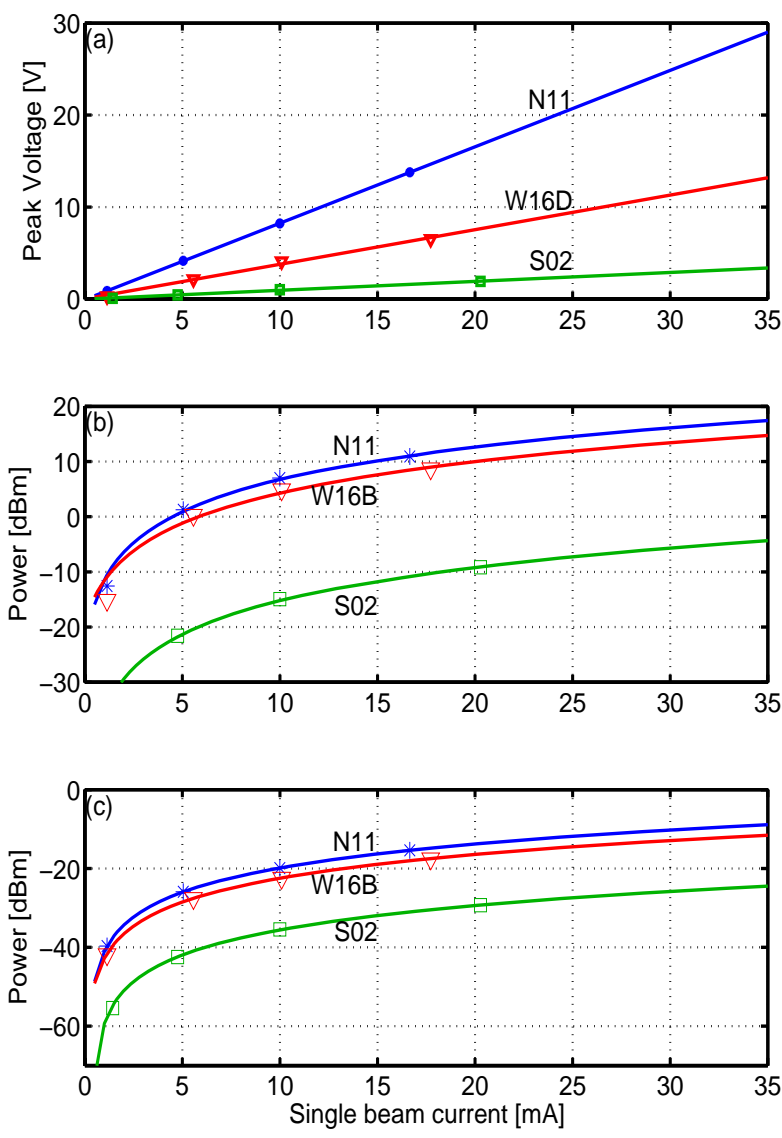

Figure 2: BPM signal strength as a function of singlebunch current. (a) The peak voltage of the BPM pickup signal (time domain) after a $1 \mathrm{GHz}$ LPF; (b) the total power input into the BPM electronics; (c) the power in the operating frequency range of $178.55 \pm 20 \mathrm{MHz}$.

Table 1: BPM signal for $I_{\text {beam }}=100 \mathrm{~mA}$. The peak voltage is after $1 \mathrm{GHz} \mathrm{LPF}$.

\begin{tabular}{lccc}
\hline \hline & N11 & W16 & S02 \\
\hline Peak Voltage [V] & 83.0 & 37.6 & 9.7 \\
Total Power [dBm] & 448.0 & 236.0 & 3.0 \\
Power @ 178.55 MHz [dBm] & 1.06 & 0.57 & 0.03 \\
\hline \hline
\end{tabular}

The BPM signals are also measured in the time and frequency domain. The time domain signal is processed in the folowing way: 1) removing the high frequency components above $1 \mathrm{GHz}$; 2) fitting the peak voltage of the pro06 Instrumentation, Controls, Feedback \& Operational Aspects cessed signal as a function of single-bunch beam current. In the frequency domain, the total input power and power in the operating frequency range are also fit as a function of single-bunch beam current, see Fig. 2. By extending the fit curves, the expected BPM signal strength is then extrapolated and summarized in Table 1. For all three types of BPMs, the peak voltage into the BPM electronics after the $1 \mathrm{GHz} \mathrm{LPF}$ is well above the 5 volts limit.

\section{BPM SIGNAL CONDITIONING FOR LOWER PEAK VOLTAGE}

The peak voltage can be reduced by applying broadband attenuators, band-pass filters (BPF) and LPFs. The attenuator attenuates all the components in frequency domain and is not suitable for working over a wide range of beam current. For selecting proper filters, we studied the spectrum of the BPM signal (see Fig. 3) which shows that the signal power is distributed in a range from first harmonic of revolution frequency, $f_{0}=2.78989 \mathrm{MHz}$, to $4 \mathrm{GHz}$ and only a very small portion of it is used by the BPM electronics. So it is possible to reduce the peak voltage by using LPF while minimize the signal loss at the operating frequency.

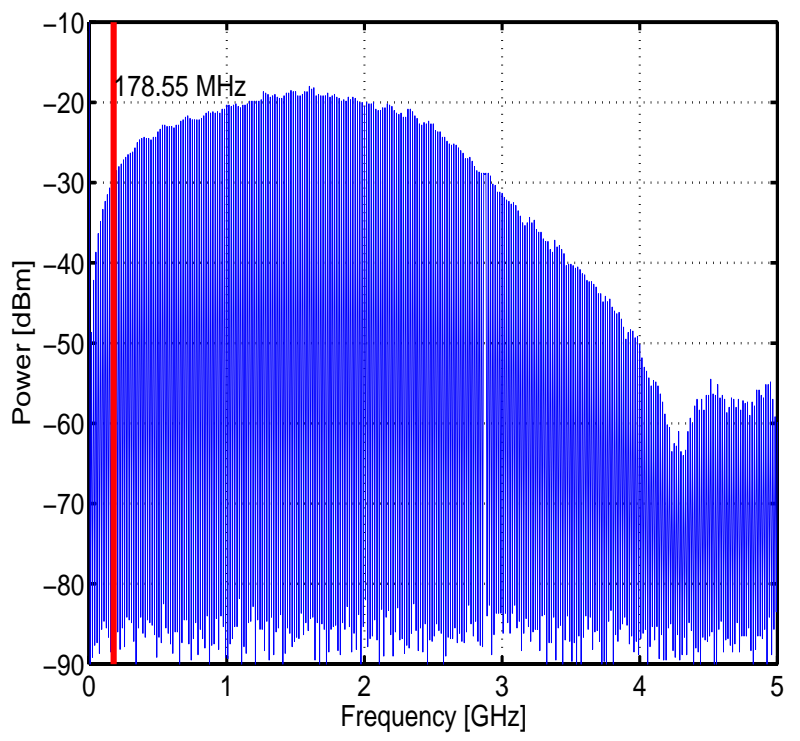

Figure 3: Signal spectrum of the signal mesured by a short stripline BPM (W16 BPM) at single bunch $I_{\text {beam }}=17.73$ $\mathrm{mA}$.

Several types of LPFs, including Mini-circuits SLP-200, VLF-320 and VLF-160+, were tested on each type pickup. By using VLF160+ (160 MHz LPF) on stripline BPMs, the storage ring can be operated from $0.5 \mathrm{~mA}$ to $100 \mathrm{~mA}$ single-bunch current (see Fig. 4 and Table 2). The orbit variation is much more reduced; from $32 \mathrm{~mA}$ to $5 \mathrm{~mA}$, the horizontal orbit variation is about $12 \mu \mathrm{m}$, the vertical orbit variation is $4 \mu \mathrm{m}$ (see Fig. 5). VLF-320 (320 MHz LPF) is suitable for button BPMs. The SLP-200 (200 MHz LPF) can not be used due to its low power handling.

T03 Beam Diagnostics and Instrumentation 

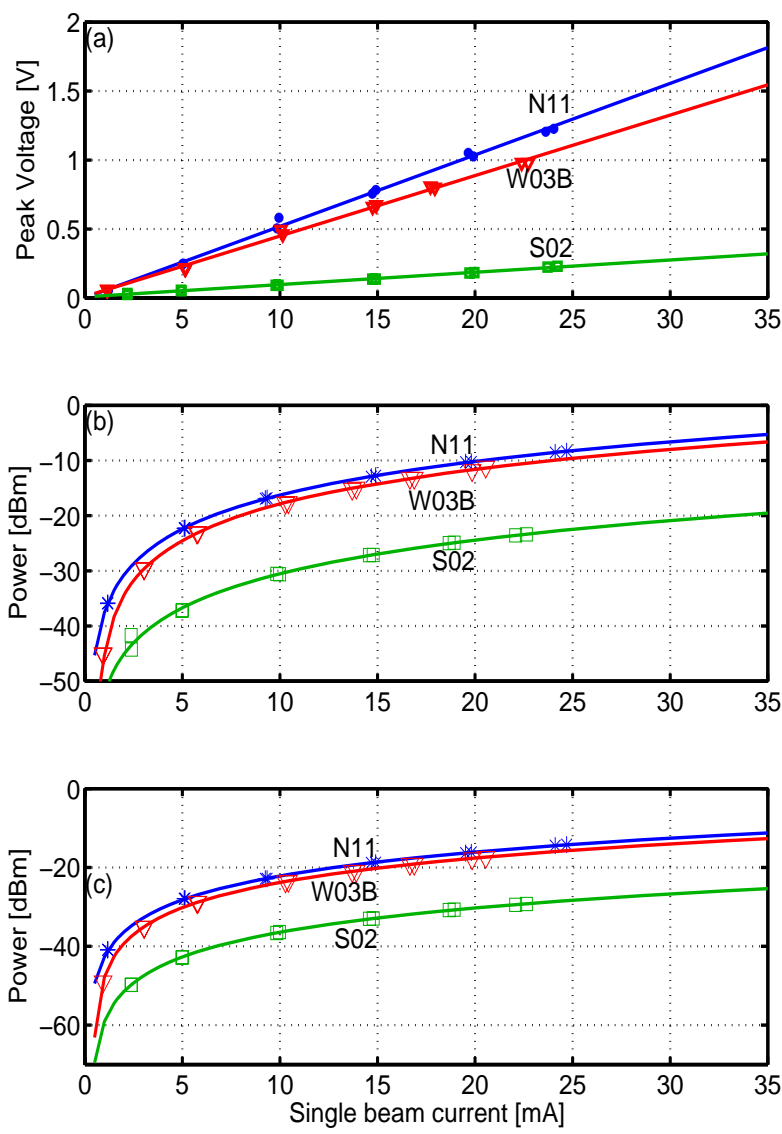

Figure 4: BPM signal after the $160 \mathrm{MHz}$ LPF as a function of single-bunch current. (a) The peak voltage of the BPM signal; (b) the total power input into the BPM electronics; (c) the power over operating frequency range 178.55 \pm 20 $\mathrm{MHz}$.

Table 2: BPM signal after $160 \mathrm{MHz}$ LPF for $I_{\text {beam }}=100$ $\mathrm{mA}$.

\begin{tabular}{lccc}
\hline \hline & N11 & W16 & S02 \\
\hline Peak Voltage [V] & 5.18 & 4.39 & 0.90 \\
Total Power [dBm] & 2.43 & 1.82 & 0.09 \\
Power @ $178.55 \mathrm{MHz}[\mathrm{dBm}]$ & 0.62 & 0.45 & 0.02 \\
\hline \hline
\end{tabular}

\section{SUMMARY}

The Mini-circuits LPF (VLF-160+ and VLF320) will be used in the Duke storage ring to effectively reduce the peak voltage while maintaining the useful signal for the BPM electronics.

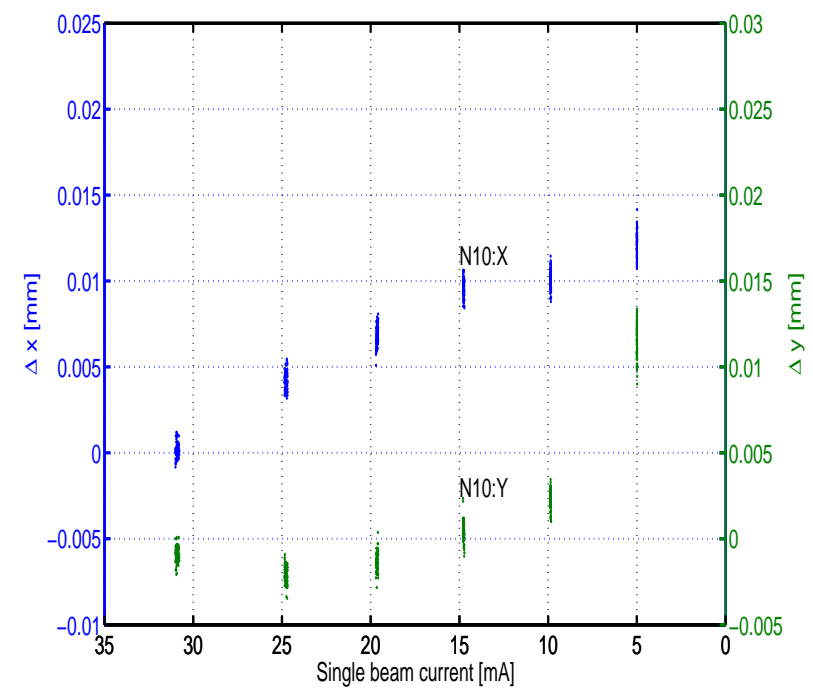

Figure 5: Beam position measured by a long stripline BPM (N10) without orbit feedback.

\section{REFERENCES}

[1] P. Wang, et al, Proc. of PAC1999, New York, P. 2099(1999).

[2] Ping Wang, private communication with Bergoz.

[3] "Multiplexed Beam Position Monitor User's Manual", http://www.bergoz.com/products/ MX-BPM/MX-BPMdownloads/files/MX-BPM.manual.1.5.4.PDF

[4] Y.K. Wu, et al, Proc. of PAC2003, Portland, Oregon, P. 2479 (2003).

[5] S. F. Mikhailov, et al, "Commissioning of the Booster Injector Synchrotron for the HIGS Facility at Duke University", these proceedings, PAC2007. 